\title{
Spectral Distortion in Lossy Compression of Hyperspectral Data
}

\author{
Bruno Aiazzi, ${ }^{1}$ Luciano Alparone, ${ }^{2}$ Stefano Baronti, ${ }^{1}$ Cinzia Lastri, ${ }^{1}$ and Massimo Selva ${ }^{1}$ \\ ${ }^{1}$ IFAC-CNR, Institute of Applied Physics "Nello Carrara", National Research Council of Italy, Via Madonna del Piano 10, \\ 50019 Sesto Fiorentino, Italy \\ ${ }^{2}$ Department Electronics \& Telecommunications, University of Florence, Via S. Marta 3, 50139 Florence, Italy \\ Correspondence should be addressed to Luciano Alparone, alparone@lci.det.unifi.it
}

Received 1 August 2011; Accepted 25 October 2011

Academic Editor: Raffaele Vitulli

Copyright () 2012 Bruno Aiazzi et al. This is an open access article distributed under the Creative Commons Attribution License, which permits unrestricted use, distribution, and reproduction in any medium, provided the original work is properly cited.

\begin{abstract}
Distortion allocation varying with wavelength in lossy compression of hyperspectral imagery is investigated, with the aim of minimizing the spectral distortion between original and decompressed data. The absolute angular error, or spectral angle mapper (SAM), is used to quantify spectral distortion, while radiometric distortions are measured by maximum absolute deviation (MAD) for near-lossless methods, for example, differential pulse code modulation (DPCM), or mean-squared error (MSE) for lossy methods, for example, spectral decorrelation followed by JPEG 2000. Two strategies of interband distortion allocation are compared: given a target average bit rate, distortion may be set to be constant with wavelength. Otherwise, it may be allocated proportionally to the noise level of each band, according to the virtually lossless protocol. Comparisons with the uncompressed originals show that the average SAM of radiance spectra is minimized by constant distortion allocation to radiance data. However, variable distortion allocation according to the virtually lossless protocol yields significantly lower SAM in case of reflectance spectra obtained from compressed radiance data, if compared with the constant distortion allocation at the same compression ratio.
\end{abstract}

\section{Introduction}

Hyperspectral imaging has dramatically changed the rationale of remote sensing of the Earth relying on spectral diversity. Rather than a sequence of spectral bands, each imaging the observed scene in a continuous interval of wavelengths of the electromagnetic radiation, a hyperspectral image is a collection of spectra, each measuring the electromagnetic properties of a pixel, that is, of a portion of the imaged scene, as shown in Figure 1. Under this perspective a hyperspectral pixel may be regarded as a sampled version of the continuous electromagnetic spectrum of the material, or mixture of materials, which is present in the elementary footprint of the instrument. Alternatively, a pixel spectrum is an element of a vector space, whose geometrical dimension is equal to the number of available spectral bands. This new approach has opened new horizons to remote sensing applications traditionally performed by photo interpreters.

Since technological advances in imaging spectrometry have led to acquisition of data that exhibit extremely high spatial, spectral, and radiometric resolution, the definition of quality of the data is changed. The quality of a multispectral image may be related to its perceivable features, like sharpness, regarded as geometrical, and color contrast. All instrument impairments, like noise and aliasing, and structured artifacts, in general, should be kept below the level of human perception. Conversely, the quality of a hyperspectral image lies in its potentiality of discriminating different materials having similar spectra. This requirement implies that pixel spectra should be enough finely sampled and clean from noise to capture small emission/absorption phenomena occurring within the scene. Thus, a valuable hyperspectral image should exhibit a high spectral resolution, at least in an interval of wavelengths of interest, and especially a high signal-to-noise ratio (SNR) [1].

When the hyperspectral imaging instrument is placed on a satellite, data compression is crucial. To meet the quality issues of hyperspectral imaging, differential pulse code modulation (DPCM) is usually employed for either lossless or near-lossless data compression. The latter indicates that the decompressed data have a user-defined maximum absolute error, being zero in the lossless case. Several variants exist in prediction schemes, the most performing being adaptive [2-6]. Lossless compression thoroughly preserves the information of the data but allows a moderate decrement 


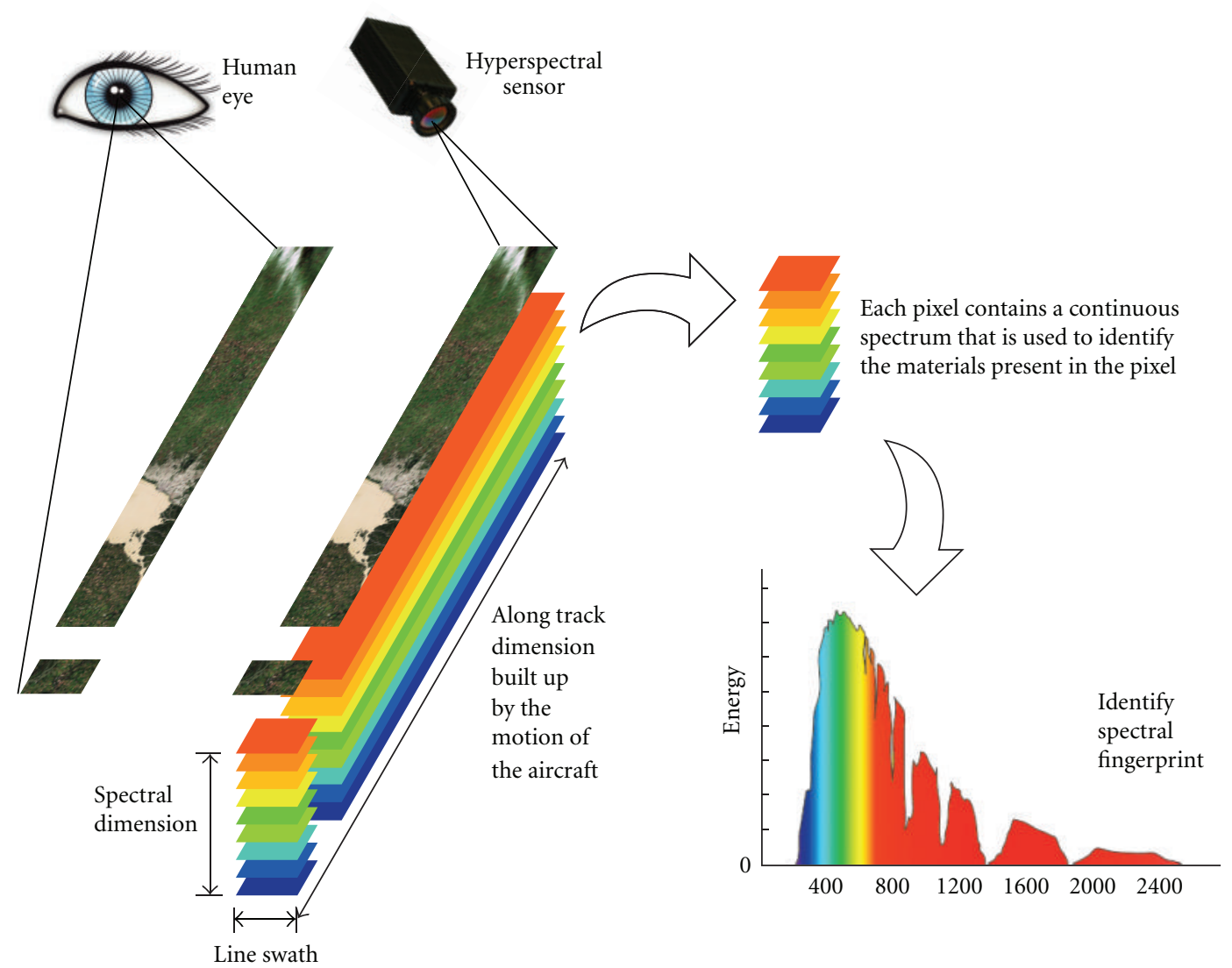

FIGURE 1: Hyperspectral remote sensing of the earth.

in transmission bit rate to be achieved. Compression ratios attained even by the most advanced schemes are lower than three, with respect to PCM coding of raw data [7-9]. Thus, the bottleneck of downlink to ground stations may severely hamper the coverage capabilities of modern satellite instruments. If strictly lossless techniques are not employed, a certain amount of information of the data will be lost. However, such statistical information may be mostly due to random fluctuations of the instrumental noise. The rationale that compression-induced distortion is more tolerable, that is, less harmful, in those bands, in which the noise is higher, constitutes the virtually lossless paradigm $[10,11]$.

In the literature, there exist several distortion measurements, some of which are suitable for quality assessment of decompressed hyperspectral data: mean square error (MSE), maximum absolute deviation (MAD), a.k.a. peak error, and SNR. The problem is that they measure the distortion introduced in the data, but cannot measure the consequences of such a distortion, that is, how the information loss would affect the outcome of an analysis performed on the data [12]. In applications of hyperspectral remote sensing, average and maximum angles between original and decompressed pixel vectors are usually adopted to measure the dissimilarity of spectra belonging to different materials. Under this perspective, spectral angle might be useful to measure the distortion of lossy compressed hyperspectral data. The main advantage of distortion metrics like spectral angle and spectral information divergence [13] is their insensitiveness to spatial variability of spectral signatures, and hence, their suitability for identification/classification of materials from their reflectance spectra. As a matter of fact, discrimination of materials is one of the most challenging task, in which hyperspectral data reveal their full potentiality. In fact, if remote sensing imagery is analyzed with the goal of recognizing broad classes of land cover, like vegetation, bare soil, urban, ice, and so forth, also data acquired by multispectral instruments are effective. Instead, if more specific tasks are concerned, such as minerals identification or geological inspections, especially on coastal waters, in order to identify the presence of chlorophyll, phytoplankton, or dissolved organic materials, the high spectral resolution captured by hyperspectral instruments is beneficial.

\section{Satellite Hyperspectral Processing Chain}

Since the pioneering mission Hyperion launched in 2001 [14] has opened new possibilities of global Earth coverage, hyperspectral imaging from a satellite platform has progressively grown in interest up to motivate the upcoming missions PRISMA [15] and EnMAP [16, 17].

The hyperspectral processing chain consists of three segments: satellite segment, ground segment, and user segment. Figure 2 highlights the main tasks performed by each segment. The on-board instruments produce data in raw 


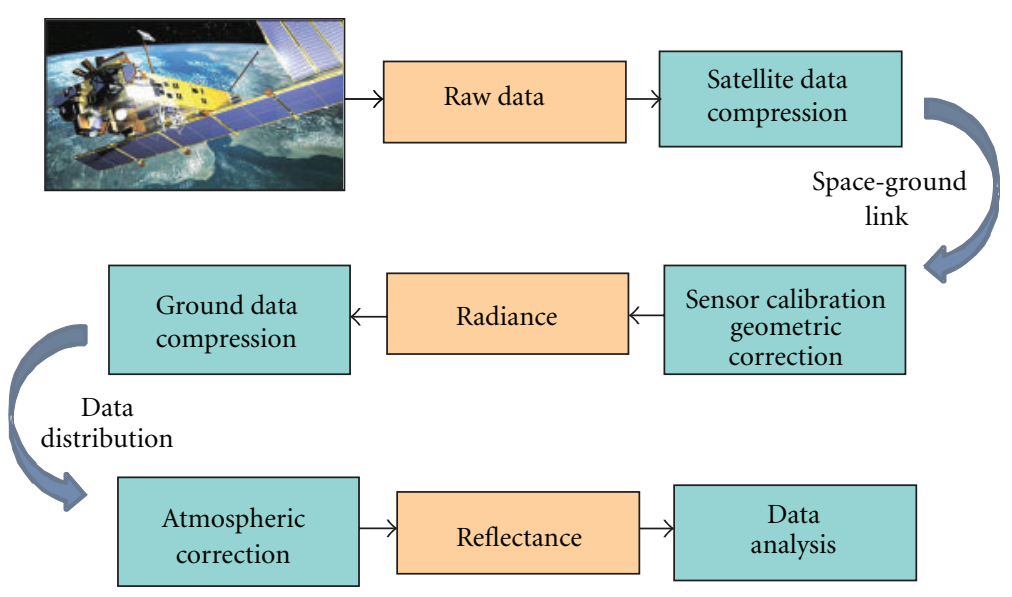

FIGURE 2: Flowchart of satellite hyperspectral processing chain. Compressed data are decoded before successive processing.

format. Raw data are expressed by means of digital counts from the analog-to-digital converter (ADC), and their value is diminished by the dark signal averaged in time before the acquisition. Raw data are compressed, with or without loss, and downloaded to the ground station(s), where the data are decompressed, converted to radiance values, and corrected for instrumental effects (e.g., striping of push-broom sensors). The calibrated data are geometrically corrected for orbital effects, georeferenced, and possibly orthorectified. All geometric operations subsequent to calibration have little impact on the quality of data products. Eventually, data products are stored in archives, generally with highly redundant formats, for example, double precision floating point per pixel radiance value, with spectral radiance measured in $\mathrm{W} \cdot \mathrm{sr}^{-1} \cdot \mathrm{m}^{-2} \cdot \mathrm{nm}^{-1}$.

When the data are distributed to users, they are usually converted to fixed-point formats (e.g., 16 bits per component, including a sign bit). This conversion may lead to a loss of information, especially because the radiance unit in the fixed-point format is $\mu \mathrm{W} \cdot \mathrm{sr}^{-1} \cdot \mathrm{m}^{-2} \cdot \mathrm{nm}^{-1}$. A finer radiance step would be 10 times smaller and would require 20 bits instead of 16 . Fixed-point radiance data are compressed, possibly with loss, and delivered to users. After decompression of radiance data, solar irradiance and atmospheric transmittance are usually corrected by users to produce reflectance spectra that may be matched to library spectra in order to recognize and classify materials. At this step, it is important to investigate the effects of a lossy compression of radiance data in terms of changes in spectral angle with respect to reflectance spectra obtained from uncompressed radiance data. At least for spectral analysis relying on measurements of spectral angle, the impact of a lossy compression of radiance data will be more predictable if angular errors of decompressed spectra will be minimized, for a given compression ratio.

\section{Distortion Measurements}

Before discussing the virtually lossless protocol, let us review some of the most widely used distortion measurements for single-band data, that is, one band of a hyperspectral image.
3.1. Radiometric Distortion. Let $0 \leq g(x, y) \leq g_{f s}$ denote an $N$-pixel digital image, and let $\tilde{g}(x, y)$ be its possibly distorted version achieved by compressing $g(x, y)$ and decoding the outcome bit stream. Widely used distortion measurements are reported in the following.

Mean absolute error (MAE), or $L_{1}$ norm of pixel error,

$$
\operatorname{MAE}=\frac{1}{N} \sum_{x} \sum_{y}|g(x, y)-\tilde{g}(x, y)|
$$

mean squared error (MSE), or $L_{2}^{2}$,

$$
\mathrm{MSE}=\frac{1}{N} \sum_{x} \sum_{y}[g(x, y)-\tilde{g}(x, y)]^{2} ;
$$

root MSE (RMSE), or $L_{2}$,

$$
\text { RMSE }=\sqrt{\mathrm{MSE}}
$$

signal-to-noise ratio (SNR)

$$
\mathrm{SNR}_{(\mathrm{dB})}=10 \cdot \log _{10} \frac{\overline{g^{2}}}{\mathrm{MSE}+1 / 12} ;
$$

maximum absolute deviation (MAD), or peak error, or $L_{\infty}$,

$$
\operatorname{MAD}=\max _{x, y}\{|g(x, y)-\tilde{g}(x, y)|\} .
$$

In (4), MSE is incremented by the variance of the integer round-off error starting from floating values, to handle the limit case, when MSE $=0$. Thus, SNR (4) will be upper bounded by $10 \cdot \log _{10}\left(12 \cdot \overline{g^{2}}\right)$.

3.2. Spectral Distortion. Given two spectral vectors $\mathbf{v}$ and $\tilde{\mathbf{v}}$ both having $L$ components, let $\mathbf{v}=\left\{v_{1}, v_{2}, \ldots, v_{L}\right\}$ be the original spectral pixel vector $v_{z}=g_{z}(x, y)$ and $\widetilde{\mathbf{v}}=$ $\left\{\tilde{v}_{1}, \widetilde{v}_{2}, \ldots, \widetilde{v}_{L}\right\}$ its distorted version obtained after lossy compression and decompression, that is, $\tilde{v}_{z}=\tilde{g}_{z}(x, y)$. Analogously to the radiometric distortion measurements, spectral distortion measurement may be defined. 
The spectral angle mapper (SAM) denotes the absolute value of the spectral angle between the couple of vectors:

$$
\operatorname{SAM}(\mathbf{v}, \tilde{\mathbf{v}}) \triangleq \arccos \left(\frac{\langle\mathbf{v}, \tilde{\mathbf{v}}\rangle}{\|\mathbf{v}\|_{2} \cdot\|\tilde{\mathbf{v}}\|_{2}}\right),
$$

in which $\langle\cdot, \cdot\rangle$ stands for scalar product. SAM can be measured in either degrees or radians.

Alternatively, SAM (6) may be either averaged on pixel vectors, or the maximum may be taken instead, as more representative of spectral quality. Note that radiometric distortion does not necessarily imply spectral distortion. Conversely, spectral distortion is always accompanied by a radiometric distortion, which is minimal for SAM when the couple of vectors has the same Euclidean length $\left(L_{2}\right)$.

\section{The Virtually Lossless Compression Protocol}

The term virtually lossless, introduced by three of the authors in 2000 [10], indicates that the distortion originated by compression does not affect the results of analysis, either manual or automated, performed on data that have been compressed. Besides the trivial case of strictly lossless compression, which is a particular case of the virtually lossless one, the rationale is that since all data are more or less affected by noise, any compression-induced distortion should appear as a negligible additional amount of noise that is statistically independent of the intrinsic noise of the data. Furthermore, the first order distributions of compression errors should be such that the overall probability density function (PDF) of the noise corrupting the decompressed data, that is, intrinsic noise plus compression-induced noise, closely matches the noise PDF of the original data. This requirement may hold if the difference between uncompressed and decompressed data exhibits a peaked and narrow PDF without tails, as it happens for near-lossless techniques, whenever the user-defined MAD is sufficiently smaller than the standard deviation $\sigma_{n}$ of the background noise. Both MAD and $\sigma_{n}$ are intended to be expressed in either physical units, for calibrated data, or as digital counts otherwise. Therefore, noise modeling and estimation from the uncompressed data becomes a major task to accomplish a virtually lossless compression [3]. The instrumental noise may also be signal dependent, as it happens with hyperspectral images, especially for new-generation instruments [18].

The key to achieve a compression preserving the scientific quality of the data for remote sensing is represented by the following twofold recommendation.

(1) Absence of tails in the PDF of the error between uncompressed and decompressed image, in order to minimize the ratio MAD/RMSE, which is equivalent to minimizing MAD for a given RMSE.

(2) MSE has to be lower by one order of magnitude (i.e., it has to be less than 0.1 times, or more than $10 \mathrm{~dB}$ lower) with respect to the variance of background noise $\sigma_{n}^{2}$. By considering the image SNR, a distortion increase of 0.1 times implies a SNR decrement of about $0.4 \mathrm{~dB}$.
In certain cases, however, it may become mandatory to increase compression ratios above the values typical of the strictly virtually lossless protocol. To adjust the compression ratio, a real-valued positive scale factor $q$ is introduced, such that the quantization step size of the $n$th band is given by

$$
\Delta_{n}=q \cdot \sigma_{n}
$$

If $q \leq 1$, a strictly virtually lossless compression is achieved, since the compression-induced quadratic distortion is less than one tenth of the intrinsic noisiness of the data. Otherwise, if $q>1$, compression is wide-sense virtually lossless, because distortion is larger, but properly allocated among the spectral bands.

\section{Experimental Results}

5.1. Virtually Lossless Compression of Radiance Data. The set of calibrated data is a sequence collected by AVIRIS in 1997 on the Cuprite Mine, NV, test site. Both spectral radiance data and reflectance data were available in uncompressed 16-bit format. The noise standard deviation $\sigma_{n}$ of the test sequence Cuprite has been measured by means of the scatterplot method [19] and is shown in Figure 3. What immediately stands out is that the noise standard deviation distribution with the wavelength roughly follows the trend of a typical spectral irradiance of the sun, unlike the raw data where this effect does not occur. The explanation is that spectral dampers aimed at flattening the spectral response of the sun are built up within the instrument in order to avoid saturation of the 14-bit ADC [9]. Hence, not only the noise, but also the signal is spectrally constant in raw data. When raw data are calibrated, they are multiplied by bandscaling gains, roughly following the shape of solar irradiance, in order to restore the physical correspondence between pixel values and spectral radiance. Thus, the noise standard deviations are multiplied by the same weights to yield the trend in Figure 3.

Figure 4 shows quantization step sizes for three different values of $q$. To compare virtually lossless compression with a unique quantizer for the whole data cube, the step size of the latter, yielding the same compression ratio as the former, is the odd integer round-off of the geometric mean of the step sizes in (7) [20].

An experiment has been performed using JPEG2000, which is an MSE-bounded encoder. To achieve a 3D decorrelation also for JPEG2000, a spectral decorrelation (a single spectral predictor with 3 coefficients, adaptively calculated for each band) has been adopted prior to JPEG2000. This decorrelator is a simplified version of the algorithm proposed in [7]. Figure 5(a) highlights that JPEG2000 coding of spectrally decorrelated data $(\mathrm{SD}+\mathrm{J} 2 \mathrm{~K})$ is far preferable to plain JPEG2000, which is unable to remove spectral redundancy. JPEG 2000 has been run by setting the rate parameter for each band equal to the desired rate. That is equivalent to obtaining a (roughly) constant MSE on every band and an MSE variable from band to band. The simple scheme adopted for lossy compression is slightly less performing than the best transform-based techniques 


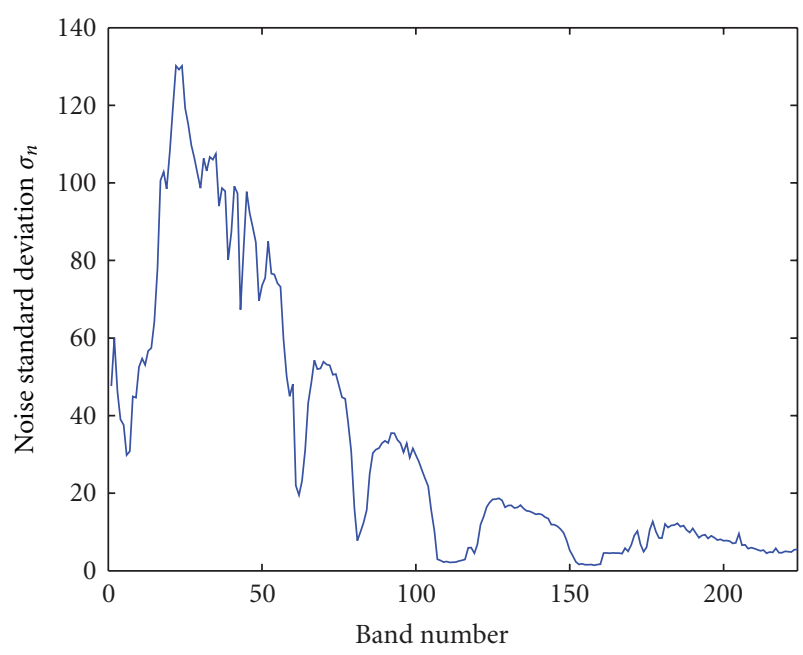

FIgure 3: Measured noise standard deviation of AVIRIS 1997 radiance data.

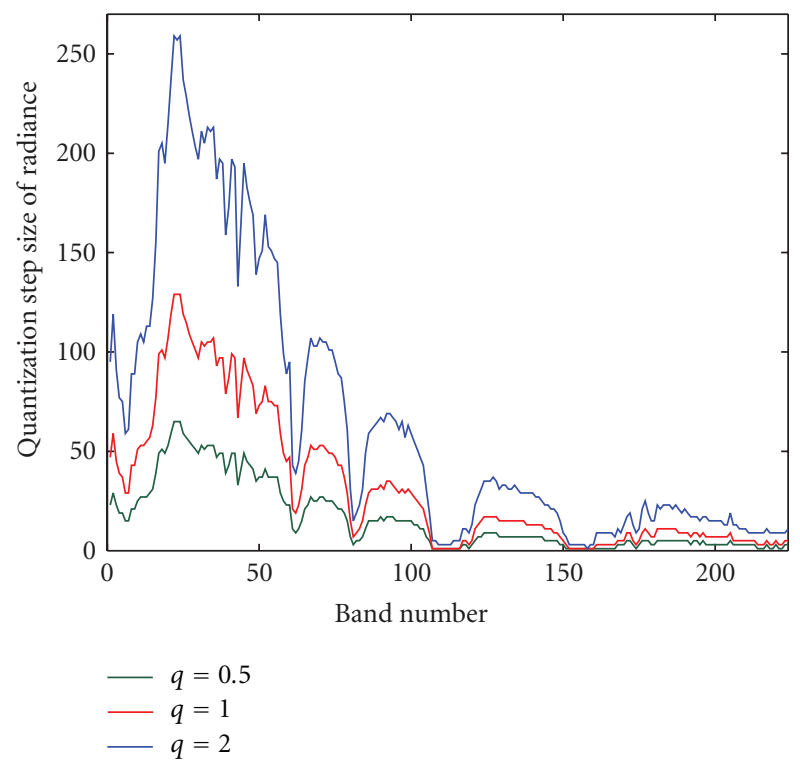

FIGURE 4: Quantization step sizes of radiance data.

recently proposed for lossy compression of hyperspectral data $[21,22]$. This fact can be inferred by considering Figure 5(a) of [7] and Figures 8 and 1 of [21, 22], respectively, which report PSNR and SNR of AVIRIS'97 Cuprite sequence. The difference in $\mathrm{dB}$ between PSNR and SNR for this data set is about $20 \mathrm{~dB}$, as can be deduced by extrapolating the representative straight line for high bit rates at the $y$-axis $(0$ bpp). The consequence is that at $1 \mathrm{bpp}$ the performances of [7] and [21, 22] are comparable. However, the goal is not to attain the ultimate compression, but to evidence what happens to spectral distortions when an MSE-bounded scheme is used in conjunction with a spectrally variable distortion allocation.

Figure 5(b) reports SAM versus bit rate for the MSEbounded encoder $(\mathrm{SD}+\mathrm{J} 2 \mathrm{~K})$. SAM is higher if distortion is

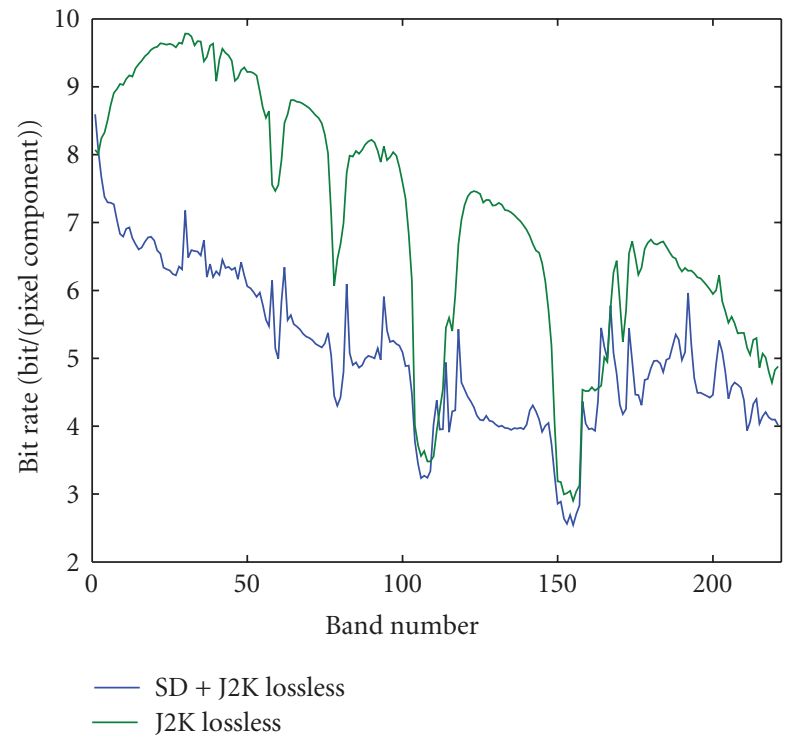

(a)

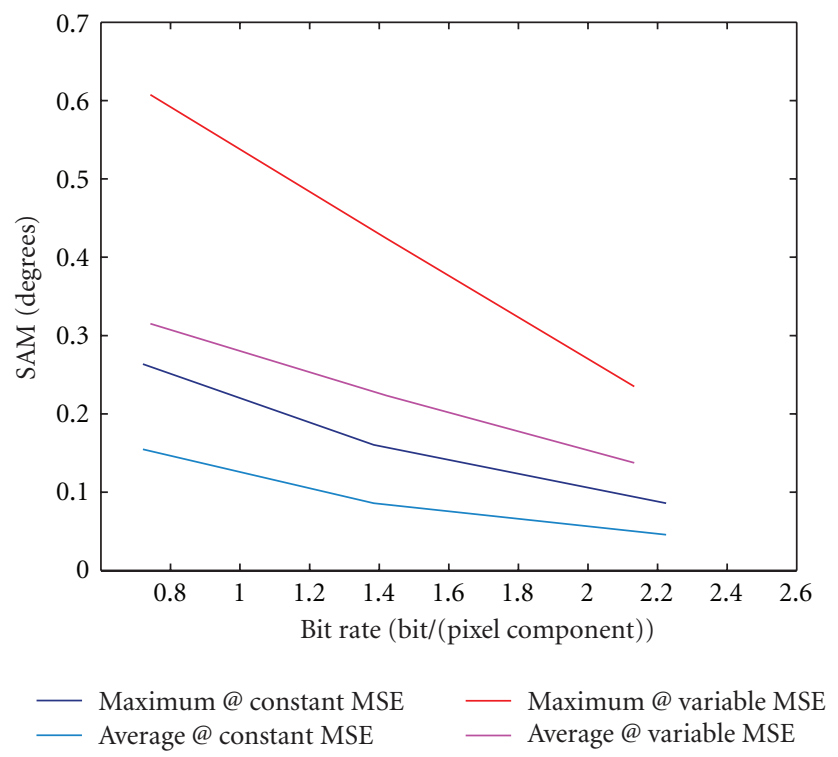

(b)

FIGURE 5: (a) Lossless compression of hyperspectral radiance data (AVIRIS'97) based on JPEG 2000, with and without spectral decorrelation; (b) SAM between original and compressed spectra varying with bit rate: MSE constant with wavelength versus MSE varying with wavelength.

allocated band by band following the noise variance. Thus, if one aims at minimizing the SAM between original and lossy compressed radiance data, the virtually lossless strategy is not recommended.

5.2. Impact of Radiance Data Compression on Reflectance Spectra. Analysis procedures of hyperspectral vectors are usually performed on reflectance data, especially when the goal is identification of materials by comparing their remotely sensed spectra with sample spectra extracted from 
reference spectral libraries. Whenever measured spectra are to be compared to laboratory spectra, the radiance data are converted into reflectance, for example, by means of the following simplified formula:

$$
\rho(\lambda)=\frac{R(\lambda) \cdot \pi}{I(\lambda) \cdot T(\lambda)},
$$

in which $\rho(\lambda)$ is the reflectance, $I(\lambda)$ is the solar irradiance on ground, $T(\lambda)$ is the atmospheric transmittance, and $R(\lambda)$ is the at-sensor radiance, all functions of wavelength $\lambda$. Distortions introduced by compression on radiance data will be amplified or attenuated depending on the values of the product $I(\lambda) \cdot T(\lambda) / \pi$. The impact of atmospheric corrections on the distortion of lossy compressed radiance data has been recently investigated [23]. The main result is that compression should better be applied to reflectance data, rather than to radiance data, according to both SNR and SAM comparisons. Unfortunately, compression of reflectance data for distribution to users is unfeasible, because inversion of the atmospheric model is a part of the application performed by users.

In the case of the AVIRIS Cuprite'97 scene, data sets for both radiance and reflectance are available. So, it is possible to recover the pointwise transfer function for conversion from radiance units to reflectance values. In this way, it is easy to evaluate how distortions introduced by compression into radiance data would affect reflectance spectra. Figure 6 shows quantization step sizes of radiance and their extrapolation to reflectance, both varying with wavelength. Compression is virtually lossless with $q=1$. Apart from absorption regions and boundary regions of the instrument, in which the equivalent step size is set to zero because it is undetermined, the trend with wavelength of reflectance step sizes is somewhat flat and quite different from the trend of radiance steps, because of (8). Analogously to Figure 4, equivalent quantization step sizes of reflectance values (rescaled to the same range as radiance values) are shown in Figure 7.

Figure 8 highlights the impact of lossy compression of radiance data on reflectance spectra, reporting SAM between spectra obtained from lossless and lossy compressed radiance data. The trends of Figure 5 are swapped, and wavelengthvariable distortion allocation provides lower SAM.

A typical application of hyperspectral remote sensing is identification of materials from measured reflectance spectra using reference spectral libraries, where reflectance spectra of hundreds of materials have been classified for reference use. SAM is usually employed to measure distances between measured and reference spectra. For each material, or class of materials, for example, clays, two thresholds may be experimentally obtained, An example is reported in Table 1. The column below the symbol "=" presents the values of angle between two spectra, such as the spectra which are actually belonging to the same material. Analogously, the symbol " $\neq$ " identifies the values of angle corresponding to definitely different materials. The column labeled with "?" reports the uncertainty interval. The thresholds in Table 1 are typical values relative to AVIRIS reflectance spectra and noise-free laboratory spectra of specimens [24]. Thus, it is expected that the average SAM error of the virtually

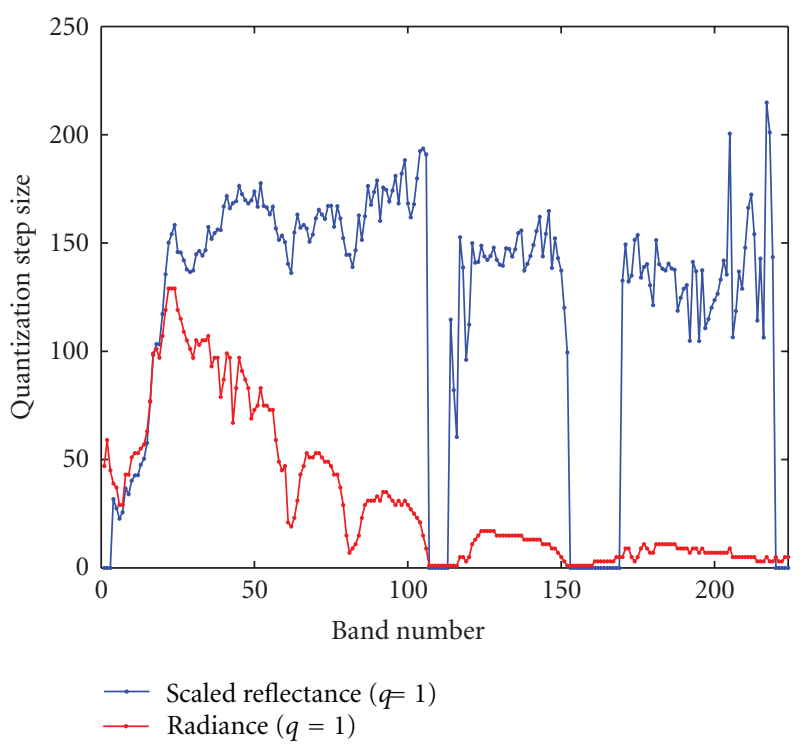

FIGURE 6: Quantization step sizes for virtually lossless compression of radiance data and their extrapolation to reflectance spectra, whose values have been rescaled in the same range.

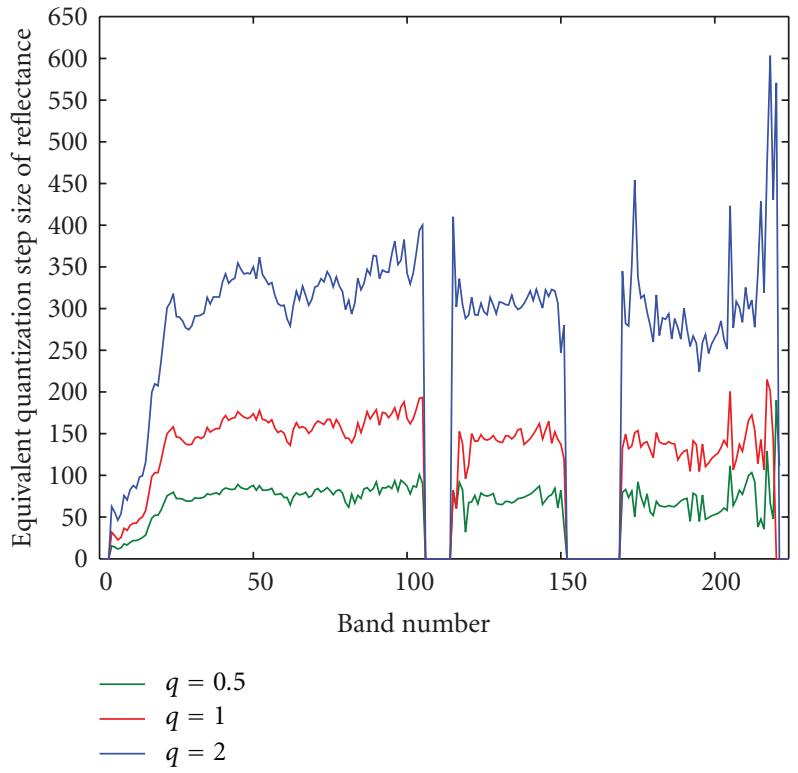

Figure 7: Equivalent quantization step sizes of reflectance values corresponding to step sizes of radiance data in Figure 4.

lossless case (0.2 degrees at 2.12 bit per pixel per band, or equivalently a 7.5 compression ratio, as shown in Figure 8) has practically no influence on the decision, because the uncertainty interval, $4^{\circ}-6^{\circ}$ in the case of uncompressed spectra, would be enlarged by $0.2^{\circ}$ in average (10\%) and by $0.4^{\circ}(20 \%)$ in the worst case, corresponding to the maximum angular error. 


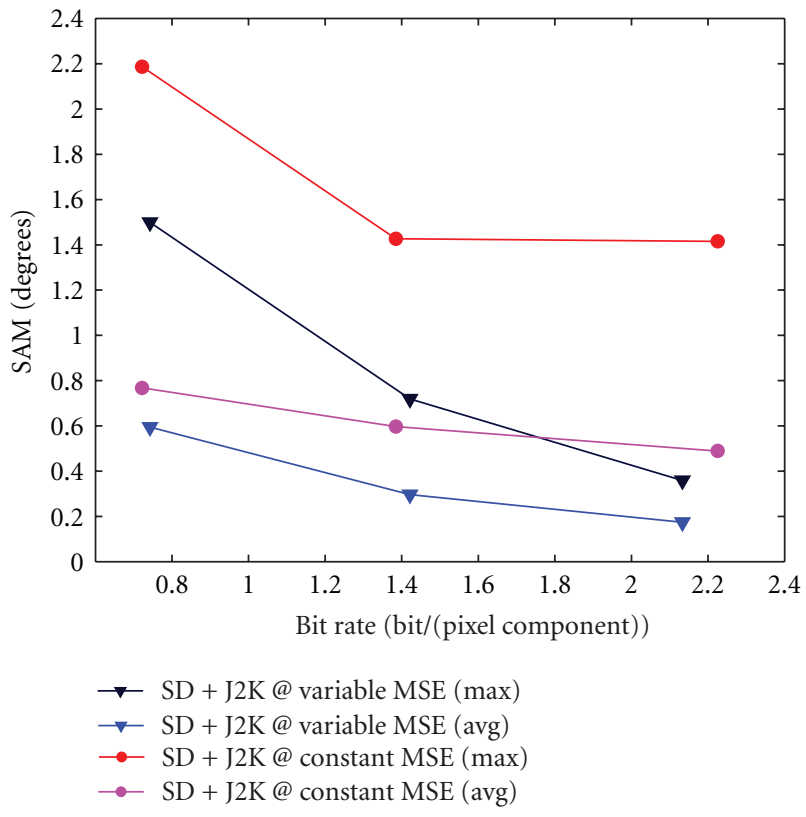

FIGURE 8: Average angular error between original reflectance spectra and those obtained from compressed radiance data plotted versus bit rate for fixed and variable MSE allocation.

TABLE 1: SAM threshold values for library spectra of clays.

\begin{tabular}{lcc}
\hline$=$ & $?$ & $\neq$ \\
\hline $\mathrm{SAM} \leq 4^{\circ}$ & $4^{\circ}<S A M \leq 6^{\circ}$ & $6^{\circ}<S A M$ \\
\hline
\end{tabular}

\section{Conclusions}

This work has highlighted that it is possible to achieve a significant reduction in the spectral angle between original and lossy compressed reflectance pixel spectra by allocating more distortion to those bands that are intrinsically noisier, when the calibrated radiance data are lossy compressed for archival and/or distribution to users. That is implicitly achieved by adopting the virtually lossless compression protocol, without affecting the average target bit rate or compression ratio. The rationale is that the SAM originated from compression should be negligible, compared to the SAM deriving from spectral differences of materials, when the decompressed radiance data are converted to reflectance spectra.

\section{Acknowledgment}

This study has been cofunded by Regione Toscana (the Regional Government of Tuscany) under Project CTOTUS in the framework of POR CReO/FESR 2007-2013.

\section{References}

[1] B. Aiazzi, L. Alparone, A. Barducci, S. Baronti, and I. Pippi, "Information-theoretic assessment of sampled hyperspectral imagers," IEEE Transactions on Geoscience and Remote Sensing, vol. 39, no. 7, pp. 1447-1458, 2001.
[2] B. Aiazzi, P. Alba, L. Alparone, and S. Baronti, "Lossless compression of multi/hyper-spectral imagery based on a 3-D fuzzy prediction," IEEE Transactions on Geoscience and Remote Sensing, vol. 37, no. 5, pp. 2287-2294, 1999.

[3] B. Aiazzi, L. Alparone, and S. Baronti, "Near-lossless compression of 3-D optical data," IEEE Transactions on Geoscience and Remote Sensing, vol. 39, no. 11, pp. 2547-2557, 2001.

[4] E. Magli, G. Olmo, and E. Quacchio, "Optimized onboard lossless and near-lossless compression of hyperspectral data using CALIC," IEEE Geoscience and Remote Sensing Letters, vol. 1, no. 1, pp. 21-25, 2004.

[5] F. Rizzo, B. Carpentieri, G. Motta, and J. A. Storer, "Lowcomplexity lossless compression of Hyperspectral imagery via linear prediction," IEEE Signal Processing Letters, vol. 12, no. 2, pp. 138-141, 2005.

[6] B. Aiazzi, L. Alparone, and S. Baronti, "On-board DPCM compression of hyperspectral data," in Proceedings of the 2nd International Workshop on On-Board Payload Data Compression (ESA OBPDC '10), R. Vitulli, Ed., CNES, France, October 2010.

[7] B. Aiazzi, L. Alparone, S. Baronti, and C. Lastri, "Crisp and fuzzy adaptive spectral predictions for lossless and near-lossless compression of hyperspectral imagery," IEEE Geoscience and Remote Sensing Letters, vol. 4, no. 4, pp. 532536, 2007.

[8] B. Aiazzi, S. Baronti, and L. Alparone, "Lossless compression of hyperspectral images using multiband lookup tables," IEEE Signal Processing Letters, vol. 16, no. 6, pp. 481-484, 2009.

[9] A. B. Kiely and M. A. Klimesh, "Exploiting calibrationinduced artifacts in lossless compression of hyperspectral imagery," IEEE Transactions on Geoscience and Remote Sensing, vol. 47, no. 8, Article ID 4814516, pp. 2672-2678, 2009.

[10] B. Aiazzi, L. Alparone, and S. Baronti, "Information preserving storage of remote sensing data: virtually lossless compression of optical and SAR images," in Proceedings of the International Geoscience and Remote Sensing Symposium (IGARSS '00), pp. 2657-2659, July 2000.

[11] C. Lastri, B. Aiazzi, L. Alparone, and S. Baronti, "Virtually lossless compression of astrophysical images," Eurasip Journal on Applied Signal Processing, vol. 2005, no. 15, pp. 2521-2535, 2005.

[12] G. Carvajal, B. Penna, and E. Magli, "Unified lossy and near-lossless hyperspectral image compression based on JPEG 2000," IEEE Geoscience and Remote Sensing Letters, vol. 5, no. 4, Article ID 4656454, pp. 593-597, 2008.

[13] C. I. Chang, "An information-theoretic approach to spectral variability, similarity, and discrimination for hyperspectral image analysis," IEEE Transactions on Information Theory, vol. 46, no. 5, pp. 1927-1932, 2000.

[14] J. S. Pearlman, P. S. Barry, C. C. Segal, J. Shepanski, D. Beiso, and S. L. Carman, "Hyperion, a space-based imaging spectrometer," IEEE Transactions on Geoscience and Remote Sensing, vol. 41, no. 6, pp. 1160-1173, 2003.

[15] D. Labate, M. Ceccherini, A. Cisbani et al., "The PRISMA payload optomechanical design, a high performance instrument for a new hyperspectral mission," Acta Astronautica, vol. 65, no. 9-10, pp. 1429-1436, 2009.

[16] L. Guanter, K. Segl, and H. Kaufmann, "Simulation of optical remote-sensing scenes with application to the EnMAP hyperspectral mission," IEEE Transactions on Geoscience and Remote Sensing, vol. 47, no. 7, Article ID 4814515, pp. 23402351, 2009.

[17] K. Segl, L. Guanter, H. Kaufmann et al., "Simulation of spatial sensor characteristics in the context of the EnMAP 
hyperspectral mission," IEEE Transactions on Geoscience and Remote Sensing, vol. 48, no. 7, Article ID 5446369, pp. 30463054, 2010.

[18] L. Alparone, M. Selva, L. Capobianco, S. Moretti, L. Chiarantini, and F. Butera, "Quality assessment of data products from a new generation airborne imaging spectrometer," in Proceedings of the IEEE International Geoscience and Remote Sensing Symposium (IGARSS '09), vol. 4, pp. 422-425, Cape Town, South Africa, July 2009.

[19] B. Aiazzi, L. Alparone, A. Barducci et al., "Noise modelling and estimation of hyperspectral data from airborne imaging spectrometers," Annals of Geophysics, vol. 49, no. 1, pp. 1-9, 2006.

[20] N. S. Jayant and P. Noll, Digital Coding of Waveforms: Principles and Applications to Speech and Video, Prentice Hall, Englewood Cliffs, NJ, USA, 1984.

[21] B. Penna, T. Tillo, E. Magli, and G. Olmo, "Transform coding techniques for lossy hyperspectral data compression,” IEEE Transactions on Geoscience and Remote Sensing, vol. 45, no. 5, pp. 1408-1421, 2007.

[22] Q. Du and J. E. Fowler, "Hyperspectral image compression using JPEG2000 and principal component analysis," IEEE Geoscience and Remote Sensing Letters, vol. 4, no. 2, pp. 201205, 2007.

[23] Q. Du, J. E. Fowler, and W. Zhu, "On the impact of atmospheric correction on lossy compression of multispectral and hyperspectral imagery," IEEE Transactions on Geoscience and Remote Sensing, vol. 47, no. 1, Article ID 4694064, pp. 130 132, 2009.

[24] A. M. Filippi and R. Archibald, "Support Vector machinebased endmember extraction," IEEE Transactions on Geoscience and Remote Sensing, vol. 47, no. 3, Article ID 4696010, pp. 771-791, 2009. 

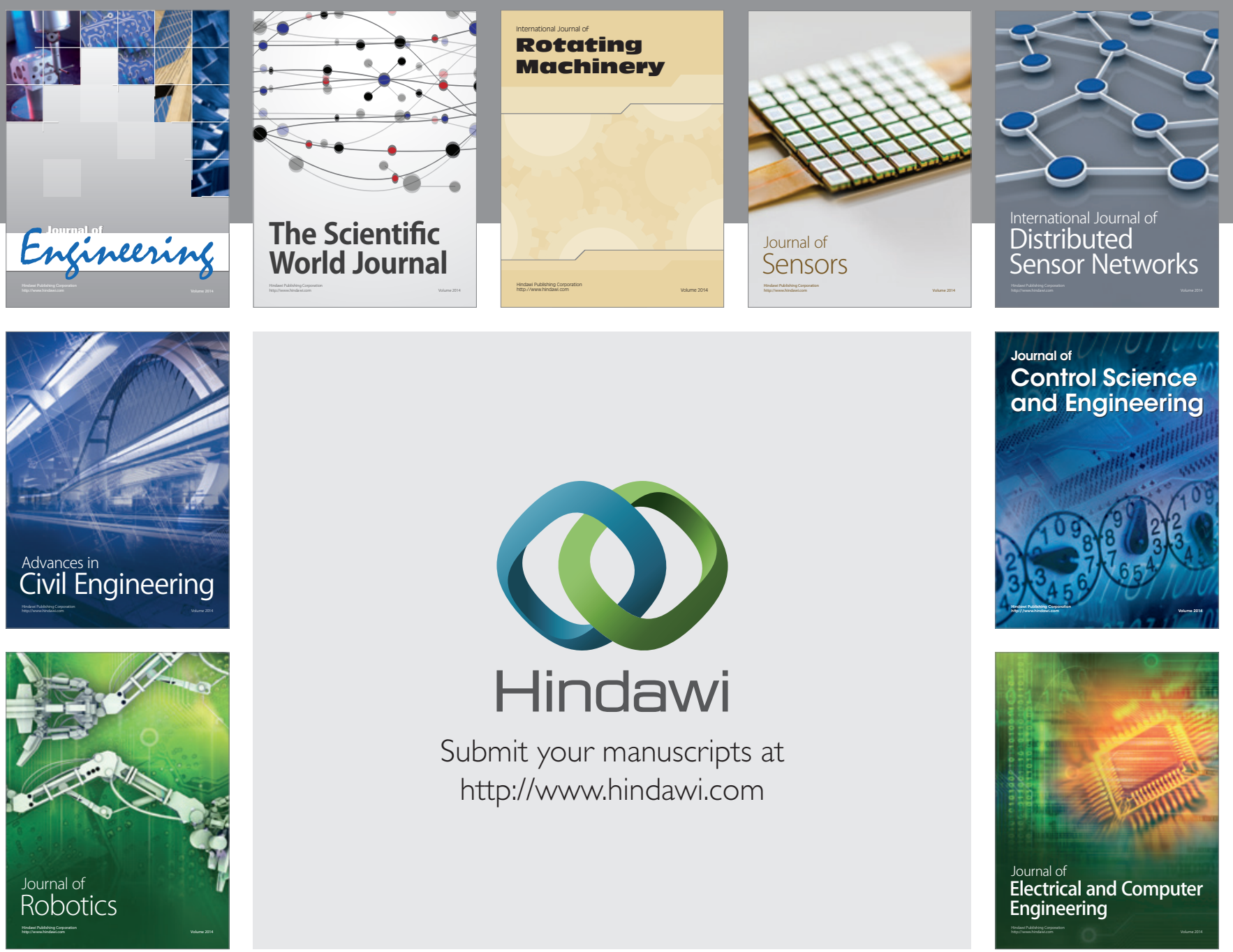

Submit your manuscripts at

http://www.hindawi.com
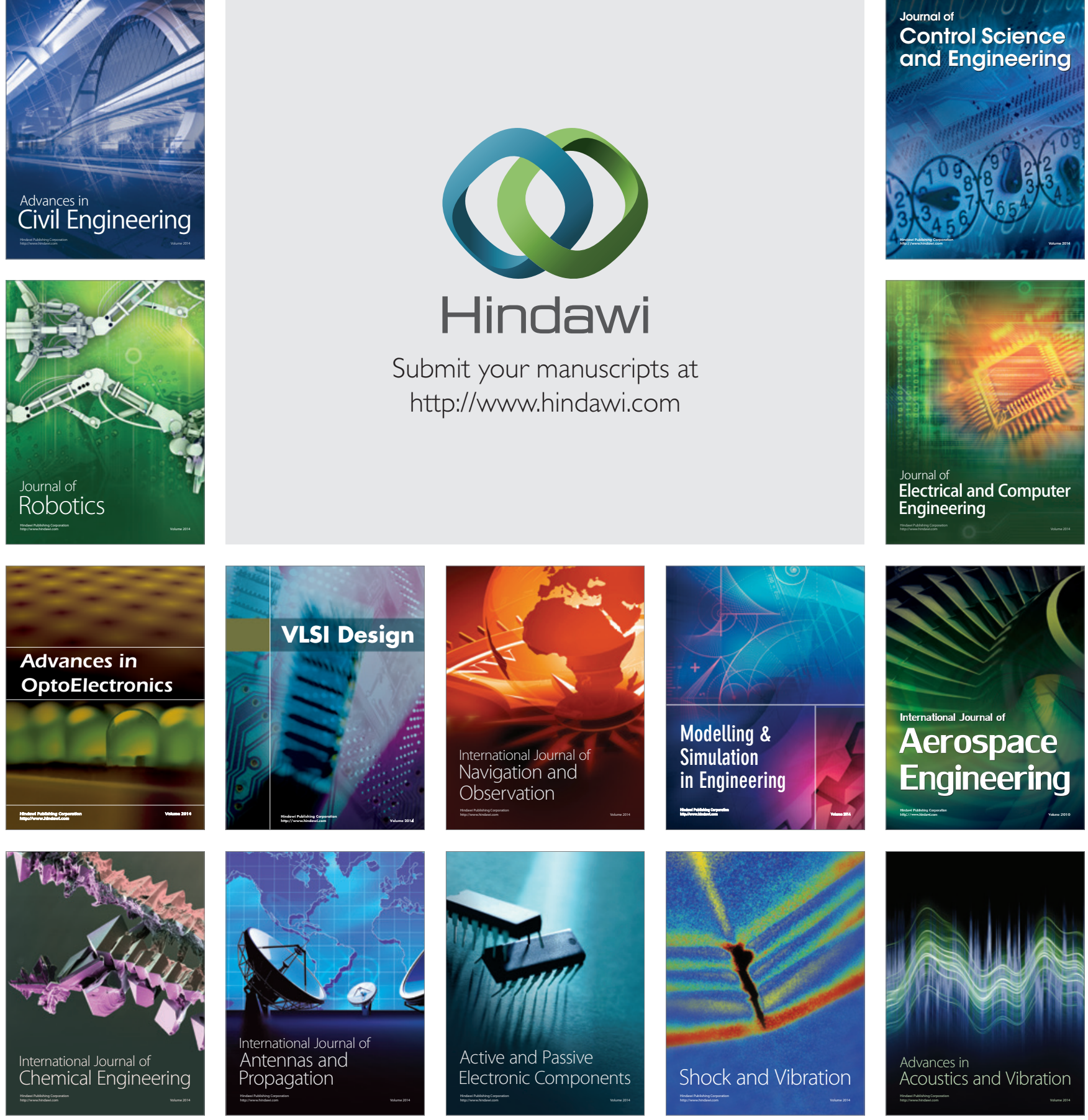